\title{
Next-generation sequencing reveals lymph node metastasis associated genetic markers in colorectal cancer
}

\author{
NI XIE ${ }^{1}$, YUJIANG YAO ${ }^{1}$, LILI WAN ${ }^{1}$, TING ZHU $^{1}$, LITAO LIU $^{1}$ and JIANHUI YUAN ${ }^{2}$ \\ ${ }^{1}$ Science and Education Administration Department of Shenzhen Second People's Hospital, \\ Shenzhen, Guangdong 518000; ${ }^{2}$ Science and Education Administration Department of Shenzhen \\ Center for Disease Control and Prevention, Shenzhen, Guangdong 518055, P.R. China
}

Received July 23, 2015; Accepted January 20, 2017

DOI: $10.3892 /$ etm.2017.4464

\begin{abstract}
Colorectal cancer is the third most prevalent type of cancer in the United States. Early diagnosis of lymph node metastases is essential to improve the prognosis for patients with colorectal cancer. Therefore, the present study aimed to screen genetic markers, including single nucleotide polymorphisms (SNPs), copy number variations (CNVs) and mRNA expression, associated with lymph node metastases in patients with colorectal cancer to enable an early diagnosis. Targeted next-generation sequencing was applied to capture SNPs and CNVs in tumor-related candidate genes within tumor tissues from 39 colorectal cancer patients; reverse transcription-quantitative polymerase chain reaction was used to detect the specific mRNA level of tumor-related candidate genes, including vascular endothelial growth factor $\mathrm{C}$, cyclin-A2, Interleukin-2, ATP-binding cassette sub-family $\mathrm{G}$ member 2, epidermal growth factor (EGF) and nuclear factor kappa B subunit 1 (NFKB1) on chromosome 4. The SNPs in solute carrier family 28 member 3 (SLC28A3), breast cancer 1 (BRCA1), ribonucleotide reductase regulators subunit M2 (RRM2), PMS1 homolog 2 (PMS2), cytidine deaminase (CDA), epoxide hydrolase 1 (EPHX1), heterogenous ribonucleoprotein particle-associated with lethal yellow (RALY), Siglec-3 (CD33), B cell lymphoma 10 (BCL10), ETS variant 1 (ETV1), macrophage stimulating 1 receptor 1 (MST1R), lysine methyltransferase 2B (KMT2B), B cell lymphoma 2 (BCL2), U6 small nuclear RNA-associated Sm-like protein 3 (LSM3), thyroid transcription factor 1 (TTF1) and mitogen-activated protein 3 kinase 1 (MAP3K1) were significantly associated with lymphatic metastasis $(\mathrm{P}<0.05)$. EGF and NFKB1 were both observed to be significantly downregulated in the lymph
\end{abstract}

Correspondence to: Professor Jianhui Yuan, Science and Education Administration Department of Shenzhen Center for Disease Control and Prevention, 8 Longyuan Road, Shenzhen, Guangdong 518055, P.R. China

E-mail: jianhui_yuan@126.com

Key words: copy number variation, single nucleotide polymorphisms, colorectal cancer, lymph node metastasis node metastases group $(\mathrm{P}<0.05)$. Although no association between CNVs and lymph node metastases in patients with colorectal cancer was observed in the present study, SNPs in SLC28A3, BRCA1, RRM2, PMS2, CDA, EPHX1, RALY, CD33, BCL10, ETV1, MST1R, KMT2B, BCL2, LSM3, TTF1 and MAP3K1 were significantly associated with colorectal cancer. Downregulation of EGF and NFKB1 was also identified to be associated with lymph node metastases in colorectal cancer. The findings of the current study provide a scientific basis for the clinical inspection of lymphatic metastasis and prognosis prediction, intervention and guidance therapy for patients with colorectal cancer.

\section{Introduction}

Malignant tumors are a life-threatening disease globally and in China. In 2015, 25\% of total mortalities were caused by cancer (1). The morbidity and mortality of cancer has been increasing for a number of years. In 2009, colorectal cancer was the cause of $8 \%$ of total mortalities caused by cancer (2). Furthermore, colorectal cancer is one of the most common tumor types. According to data published by the National Cancer Institute in 2016, colorectal cancer was the third most common tumor type (1). In developing countries, the rate of colorectal cancer is also growing rapidly. From 2010-2012 developing countries contributed to $52 \%$ of the total number of mortalities caused by colorectal cancer, and limited medical resources meant patients had a poor prognosis and survival rate (3). Treatment for colorectal cancer remains limited to traditional methods, such as surgical operations, radiotherapies and chemotherapies.

The emerging targeted molecular therapies were gradually accepted by doctors and demonstrated particular advantages in clinical treatment for colorectal cancer (4). The combination of emerging and traditional therapies improves the level of disease-free survival, survival rate and prognosis in patients with colorectal cancer. However, disease recurrence following surgery or chemotherapy, drug resistance and deterioration remains inevitable $(5,6)$.

The occurrence and development of colorectal cancer is a process controlled by multiple genes and variable factors. For example, the migration inhibitory factor/cluster of differentiation 74 signalling axis has recently been identified 
as a novel therapeutic target for colon cancer (7). Furthermore, loss of periplakin has been demonstrated to be associated with tumorigenesis of colon cancer (8). The lymph node is the primary defence against the metastasis of colorectal tumors, and is also where deterioration of health begins in patients with colorectal cancer.

Lymph node metastasis may worsen the prognosis, reduce the survival rate and even make patients more susceptible to the recurrence of colorectal cancer (3). Furthermore, lymph node metastasis may worsen the postoperative curative effect and enhance drug-resistance to chemotherapies for colorectal cancer. Previous research based on clinical practical experience demonstrated that the 5-year survival rate may reach $60-80 \%$ in patients without lymph node metastasis; however, the 5-year survival rate of patients with lymph node metastasis may only reach $30 \%$ (9). Surgery is unable to improve the prognosis of patients with colorectal cancer.

With the development of molecular biology, the understanding of colorectal cancer has progressed beyond the cellular level and further to elucidate the role of genetic biomarkers. There are two types of genetic alterations in colorectal cancer, chromosomal instability (CIN) and microsatellite instability (MSI). Aneuploidy and polyploidy are common phenotypes in CIN and contribute to $80-85 \%$ of the morbidity of colorectal cancer (10). MSI is primarily caused by errors in the DNA repairing process, and contributes to $15-20 \%$ of the total morbidity (10). There are multiple chromosome sites with copy number variation (CNV) in CIN-type colorectal cancer (11). If CNV occurs inside or around the tumor-associated gene sequences, oncogenes may be activated and anti-oncogenes may be inactivated, which eventually induces tumorigenesis (12). A previous study indicated that increased CNVs may be associated with the progression of colitis gravis to colorectal cancer (13). Another study demonstrated that CNVs were able to determine the lymph node metastasis in colorectal cancer (14). Evidence now indicates that the occurrence of CNVs in chromosome 4 may seriously induce lymph node metastasis in colorectal cancer (14-16).

Single nucleotide polymorphisms (SNPs) are a primary cause for variation in the human race. However, the association between SNPs and colorectal cancer remains unclear. In the present study, 1,053 associated genes on chromosome 4 were screened for lymph node metastasis-associated SNPs and CNVs and the mRNA level of lymph node metastasis on these genes was further investigated. The current study aimed to provide a molecular basis for clinical tests and treatment.

\section{Materials and methods}

Subjects. A total of 78 tissue samples (39 colorectal tumor and 39 normal tissues) from 39 patients were collected between January 2013 and September 2014 following tumor reduction surgery in Shenzhen Second People's Hospital (Shenzhen, China). The collection of tissues was approved by the Ethics Committee of Shenzhen Second People's Hospital. Written informed consent was provided by all patients. All experiments using human blood samples were conducted in accordance with the Clinical Sample Collection and Treatment Guidelines outlined by the Ethics Committee of Shenzen Second
People's Hospital. Among the 39 patients, 19 were female and 20 were male. The age of the subjects ranged from 29-84, with a mean age of 61.4 years. There were 19 cases with tumor diameters $\leq 5 \mathrm{~cm}$ and 20 cases with tumor diameters $>5 \mathrm{~cm}$. In the 39 patients, 29 exhibited tumors in the colon, whereas 10 patients exhibited tumors in the rectum. There were 6 cases with highly-differentiated tumors, 25 with medium-differentiated tumors and 8 with low-differentiated tumors, according to a differentiation scale determined by the Pathology Department of Shenzhen Second People's Hospital. There were 17 cases presenting lymph node metastasis, 8 cases were identified with distant metastasis and 14 cases exhibited no tumor metastasis. Pathological staging demonstrated that 19 cases were at phase I-II and 20 cases were at phase III-IV. Pathological staging was assessed according to the TNM colorectal cancer staging system of the American Joint Committee on Cancer (AJCC; AJCC staging system 7th edition 2011; cancerstaging.org) and the Union for International Cancer Control (uicc.org) standards. All patients included in the study were tested to exclude other diseases, such as gastroenteritis.

DNA and RNA extraction. During surgery, $0.2 \mathrm{~g}$ tissue was harvested from each patient, pre-treated with liquid nitrogen and processed into a powder. The DNA extraction procedure was performed using a DNeasy Blood \& Tissue kit (Qiagen $\mathrm{GmbH}$, Hilden, Germany), according to the manufacturer's instructions. RNA extraction was performed using TRIzol reagent (Ambion; Thermo Fisher Scientific, Inc., Waltham, MA, USA), following the manufacturer's instructions. The nucleotide concentration was determined using a Qubit 3.0 fluorometer and the integrity of DNA molecules were examined by electrophoresis, using $1 \%$ agarose gel stained with $0.01 \%$ acridine orange.

Exosome sequencing and bioinformatics analysis. The exosome sequencing was conducted using the Hiesq 2000 System (Illumina, Inc., San Diego, CA, USA) with a NimbleGen 4.6 microarray chip (Roche Diagnostics, Basel, Switzerland). The raw data were acquired and filtered according to signal intensity, gene annotation and sequence clustering. The sequences were then compared and statistically analyzed. Comparative genome hybridization $(\mathrm{CGH})$ data were analyzed using $\mathrm{R}$ 3.3.2 rCGH software obtained from Bioconductor (bioconductor.org). Log ratios of CNVs were calculated by comparing normalized data from the sequencing of tumor and normal tissues.

Gene expressionanalysisviareverse transcription-quantitative polymerase chain reaction $(R T-q P C R)$. RNA was extracted from the tumor and normal tissues, as described above. RNA samples were reverse transcribed using a PrimeScript Reverse Transcription kit (Takara Biotechnology Co., Ltd., Dalian, China), according to the manufacturer's instructions. A SYBR Premix Ex Taq kit (Takara Biotechnology Co., Ltd.) was used for qPCR, according to the manufacturer's instructions. The RT-qPCR primers for vascular endothelial growth factor C (VEGFC), cyclin-A2 (CCNA2), interleukin-2 (IL2), ATP-binding cassette sub-family G member 2 (ABCG2), epidermal growth factor (EGF), nuclear factor kappa B subunit 1 (NFKB1) and glyceraldehyde 3-phosphate 
dehydrogenase (GAPDH) are presented in Table I. Blank controls using only primers or templates were included in the RT-qPCR experiment. An Applied Biosystems 7900HT Fast Real-Time PCR machine (Applied Biosystems; Thermo Fisher Scientific, Inc.) was used to run the following reaction conditions: $95^{\circ} \mathrm{C}$ for $15 \mathrm{~min}$, then 40 cycles of $95^{\circ} \mathrm{C}$ for $10 \mathrm{sec}$ and $60^{\circ} \mathrm{C}$ for $30 \mathrm{sec}$. Relative gene expression levels were normalized to GAPDH, according to the $2^{-\Delta \Delta C q}$ method $(10,17)$. Experiments were performed in triplicate.

Statistical analysis. SPSS version 16.0 (SPSS, Inc., Chicago, IL, USA) was used to run data normalization and statistical tests. CGH data were analyzed using R 3.3.2 rCGH software obtained from Bioconductor (bioconductor.org). Fisher's exact test was applied to evaluate the association among SNPs, CNVs and lymph node metastasis in colorectal cancer. Student's t-test was applied to test the statistical significance of gene expression alteration. $\mathrm{P}<0.05$ was considered to indicate a statistically significant difference.

\section{Results}

Quality control of sequencing data. Raw data were filtered and adapters were removed. Sequencing results demonstrated that the target sequence was $4.5 \times 10^{12}$ base pairs, with coverage of $>99 \%$ and the sequencing depth of $\times 250$. A quality check was automatically performed on the raw data by the R 3.3.2 rCGH package, and the qualified data were used for subsequent analysis.

Association between SNPs and lymph node metastasis colorectal cancer. All 20,000 SNPs in the 1,053 cancer related genes were analyzed, 10,000 nonsense mutations were filtered out and the remaining SNPs were analyzed by statistical tests. Results indicated that 21 SNPs in 16 genes were significantly associated with colorectal cancer $(\mathrm{P}<0.05)$. Patients with lymph node metastasis exhibited higher mutation rates of solute carrier family 28 member 3 (SLC28A3; rs10868138, rs56350726), breast cancer 1 (BRCA1; rs16941, rs16942, rs799917, rs1799966), ribonucleotide reductase regulators subunit M2 (RRM2; rs1130609), PMS1 homolog 2 (PMS2; rs1805323), cytidine deaminase (CDA; rs2072671), epoxide hydrolase 1 (EPHX1; rs2234922), heterogeneous ribonucleoprotein particle-associated with lethal yellow (RALY; rs2281209), Siglec-3 (CD33; rs2455069), B cell lymphoma 10 (BCL10; rs3768235) and ETS variant 1 (ETV1; rs9639168) than patients without lymph node metastasis $(\mathrm{P}<0.05)$. Patients with lymph node metastasis had a lower mutation frequency of macrophage stimulating 1 receptor 1 (MST1R; rs1062633), lysine methyltransferase 2B (KMT2B; rs16970649), B cell lymphoma 2 (BCL2; rs1800477), U6 small nuclear RNA-associated Sm-like protein 3 (rs1870134), thyroid transcription factor 1 (TTF1; rs3739914, rs8999) and mitogen-activated protein 3 kinase 1 (MAP3K1; rs702689) than patients without lymph node metastasis $(\mathrm{P}<0.05)$. These results are presented in Table II.

Clustering analysis of lymph node metastasis-associated SNPs in colorectal cancer. The mutations present in patients were analyzed via hierarchical clustering. The results divided
Table I. Primer sequences for polymerase chain reaction.

\begin{tabular}{|c|c|c|}
\hline Gene & Sequence, $5^{\prime} \rightarrow 3^{\prime}$ & $\begin{array}{l}\text { Product } \\
\text { length, bp }\end{array}$ \\
\hline GAPDH & $\begin{array}{l}\text { F: GGGTGTGAACCATGAGAAGT } \\
\text { R: CAGTGATGGCATGGACTGTG }\end{array}$ & 149 \\
\hline VEGFC & $\begin{array}{l}\text { F: TGGGGAAGGAGTTTGGAGTC } \\
\text { R: GTTACTGGTTTGGGGCCTTG }\end{array}$ & 181 \\
\hline CCNA2 & $\begin{array}{l}\text { F: TGCTGACCCATACCTCAAGT } \\
\text { R: GGTAGGTCTGGTGAAGGTCC }\end{array}$ & 167 \\
\hline IL2 & $\begin{array}{l}\text { F: AACTCACCAGGATGCTCACA } \\
\text { R: TGCTGATTAAGTCCCTGGGT }\end{array}$ & 159 \\
\hline ABCG2 & $\begin{array}{l}\text { F: ACGCATCCTGAGATCCTGAG } \\
\text { R: CAGGTCATTGGAAGCTGTCG }\end{array}$ & 155 \\
\hline EGF & $\begin{array}{l}\text { F: CAGGGAAGATGACCACCACT } \\
\text { R: TCTCGGTACTGACATCGCTC }\end{array}$ & 168 \\
\hline NFKB1 & $\begin{array}{l}\text { F: TGTCCAGCTTCGGAGGAAAT } \\
\text { R: CACTACCAAACATGCCTCCG }\end{array}$ & 182 \\
\hline
\end{tabular}

F, forward sequence; R, reverse sequence; GAPDH, glyceraldehyde 3-phosphate dehydrogenase; VEGFC, vascular endothelial growth factor C; CCNA2, cyclin-A2; IL2, Interleukin-2; ABCG2, ATP-binding cassette sub-family $\mathrm{G}$ member 2; EGF, epidermal growth factor; NKFB1, nuclear factor kappa B subunit 1 .

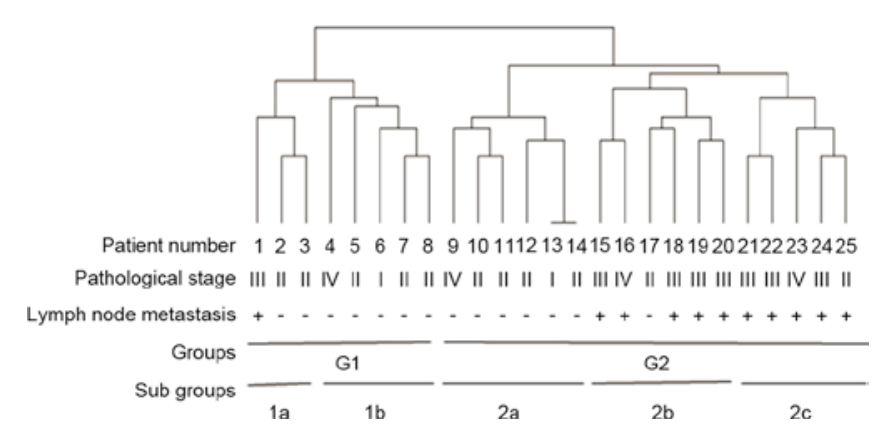

Figure 1. Hierarchical clustering of clinical data from 39 colorectal cancer patients. +, patients with lymph node metastasis; -, patients without lymph node metastasis.

patients into two groups (G1 and G2) and five sub-groups (1a, 1b, 2a, 2b and 2c). Results demonstrated an association between colorectal cancer stage and mutation frequency, with a higher rate of lymph node metastasis being present in the later stages of colorectal cancer (Fig. 1). Clustering of 21 lymph node metastasis-associated SNPs in colorectal cancer indicated that the mutation frequencies of SLC28A3, BRCA1, RRM2, PMS2, CDA and ETV1 were associated with the G2 group and the mutation frequencies of EPHX1, RALY, CD33 and BCL10 were associated with groups $2 \mathrm{~b}$ and $2 \mathrm{c}$ (Fig. 2). Multiple mutations in BRCA1 were closely clustered, indicating that BRCA1 may be a potential marker for lymph node metastasis in colon cancer. However, a larger sample size is required to verify genetic hallmarks for lymph node metastasis.

Association between $C N V s$ and lymph node metastasis in colorectal cancer. Of all cases, 15 presented with copy 
Table II. Association between SNPs and lymph node metastasis colorectal cancer.

\begin{tabular}{|c|c|c|c|c|c|}
\hline \multirow[b]{2}{*}{ Gene } & \multirow[b]{2}{*}{ SNP, rs ID } & \multirow[b]{2}{*}{ Gene type } & \multicolumn{2}{|c|}{ Lymph node metastasis, n (\%) } & \multirow[b]{2}{*}{ P-value } \\
\hline & & & $\begin{array}{c}\text { With lymph } \\
\text { node metastasis }\end{array}$ & $\begin{array}{l}\text { Without lymph } \\
\text { node metastasis }\end{array}$ & \\
\hline \multirow[t]{2}{*}{ MST1R } & rs 1062633 & $\mathrm{TC} / \mathrm{CC} \mathrm{C}$ & $3(27.3)$ & $10(71.4)$ & 0.047 \\
\hline & & $\mathrm{TT}$ & $8(72.7)$ & $4(28.6)$ & \\
\hline \multirow[t]{2}{*}{ SLC28A3 } & rs10868138 & $\mathrm{TC}$ & $6(54.5)$ & $0(0)$ & $0.003^{\mathrm{a}}$ \\
\hline & & $\mathrm{TT}$ & $5(45.5)$ & $14(100)$ & \\
\hline \multirow[t]{2}{*}{ SLC28A3 } & rs56350726 & TA/AA & 7 (63.6) & $0(0.0)$ & $0.001^{\mathrm{a}}$ \\
\hline & & $\mathrm{TT}$ & $4(36.4)$ & $14(100.0)$ & \\
\hline \multirow[t]{2}{*}{ RRM2 } & rs 1130609 & TG/GG & $8(72.7)$ & $4(28.6)$ & 0.047 \\
\hline & & TT & $3(27.3)$ & $10(71.4)$ & \\
\hline \multirow[t]{2}{*}{ BRCA1 } & rs16941 & $\mathrm{TC} / \mathrm{CC}$ & $10(90.9)$ & $7(50.0)$ & 0.042 \\
\hline & & $\mathrm{TT}$ & $1(9.1)$ & $7(50.0)$ & \\
\hline \multirow[t]{2}{*}{ BRCA1 } & rs16942 & $\mathrm{TC} / \mathrm{CC}$ & $10(90.9)$ & $7(50.0)$ & 0.042 \\
\hline & & TT & $1(9.1)$ & $7(50.0)$ & \\
\hline \multirow[t]{2}{*}{ BRCA1 } & rs799917 & GA/AA & $10(90.9)$ & $7(50.0)$ & 0.042 \\
\hline & & GG & $1(9.1)$ & $7(50.0)$ & \\
\hline \multirow[t]{2}{*}{ BRCA1 } & rs 1799966 & $\mathrm{TC} / \mathrm{CC}$ & $10(90.9)$ & $7(50.0)$ & 0.042 \\
\hline & & $\mathrm{TT}$ & $1(9.1)$ & $7(50.0)$ & \\
\hline \multirow[t]{2}{*}{ KMT2B } & rs16970649 & $\mathrm{CT}$ & $0(0)$ & $5(35.7)$ & 0.046 \\
\hline & & $\mathrm{CC}$ & $11(100)$ & $9(64.3)$ & \\
\hline \multirow[t]{2}{*}{ BCL2 } & rs 1800477 & $\mathrm{CT} / \mathrm{TT}$ & $0(0.0)$ & $5(35.7)$ & 0.046 \\
\hline & & $\mathrm{CC}$ & $11(100)$ & $9(64.3)$ & \\
\hline \multirow[t]{2}{*}{ PMS2 } & rs 1805323 & GT/TT & $10(90.9)$ & $7(50.0)$ & 0.042 \\
\hline & & GG & $1(9.1)$ & $7(50.0)$ & \\
\hline \multirow[t]{2}{*}{ LSM3 } & rs 1870134 & $\mathrm{GC} / \mathrm{CC}$ & $1(9.1)$ & $8(57.1)$ & 0.033 \\
\hline & & GG & $10(90.9)$ & $6(42.9)$ & \\
\hline \multirow[t]{2}{*}{ CDA } & rs2072671 & $\mathrm{AC} / \mathrm{CC}$ & 7 (63.6) & $3(21.4)$ & 0.049 \\
\hline & & AA & $4(36.4)$ & $11(78.6)$ & \\
\hline \multirow[t]{2}{*}{ EPHX1 } & rs2234922 & AG/GG & $4(36.4)$ & $0(0.0)$ & 0.026 \\
\hline & & AA & 7 (63.6) & $14(100.0)$ & \\
\hline \multirow[t]{2}{*}{ RALY } & rs2281209 & GA & $4(36.4)$ & $0(0.0)$ & 0.026 \\
\hline & & GG & 7 (63.6) & $14(100.0)$ & \\
\hline \multirow[t]{2}{*}{ CD33 } & rs 2455069 & $\mathrm{AG}$ & $4(36.4)$ & $0(0.0)$ & 0.026 \\
\hline & & AA & 7 (63.6) & $14(100.0)$ & \\
\hline \multirow[t]{2}{*}{ TTF1 } & rs3739914 & $\mathrm{AG} / \mathrm{GG}$ & $2(18.2)$ & $9(64.3)$ & 0.042 \\
\hline & & $\mathrm{AA}$ & $9(81.8)$ & $5(35.7)$ & \\
\hline \multirow[t]{2}{*}{ TTF1 } & rs8999 & $\mathrm{CA} / \mathrm{AA}$ & $2(18.2)$ & $10(71.4)$ & 0.015 \\
\hline & & $\mathrm{CC}$ & $9(81.8)$ & $4(28.6)$ & \\
\hline \multirow[t]{2}{*}{ BCL10 } & rs3768235 & $\mathrm{CT}$ & $6(54.5)$ & $1(7.1)$ & 0.021 \\
\hline & & $\mathrm{CC}$ & $5(45.5)$ & $13(92.9)$ & \\
\hline MAP3K1 & rs 702689 & GA/AA & $1(9.1)$ & $9(64.3)$ & 0.012 \\
\hline & & GG & $10(90.9)$ & $5(35.7)$ & \\
\hline ETV1 & rs9639168 & $\mathrm{TC} / \mathrm{CC}$ & $9(81.8)$ & $5(35.7)$ & 0.042 \\
\hline & & $\mathrm{TT}$ & $2(18.2 \%)$ & $9(64.3 \%)$ & \\
\hline
\end{tabular}

${ }^{a} \mathrm{P}<0.01$; all $\mathrm{P}<0.05$. SNP, single nucleotide polymorphism; MST1R, macrophage stimulating 1 receptor 1; SLC28A3, solute carrier family 28 member 3; RRM2, ribonucleotide reductase regulators subunit M2; BRCA1, breast cancer 1; KMT2B, lysine methyltransferase 2B; BCL2, B cell lymphoma 2; PMS2, PMS1 homolog 2; LSM3, U6 small nuclear RNA-associated Sm-like protein 3; CDA, cytidine deaminase; EPHX1, epoxide hydrolase 1; RALY, ribonucleoprotein particle-associated with lethal yellow; CD33, Siglec-3; TTF1, thyroid transcription factor 1; BCL10, B cell lymphoma 10; MAP3K1, mitogen-activated protein 3 kinase 1; ETV1, ETS variant 1. 


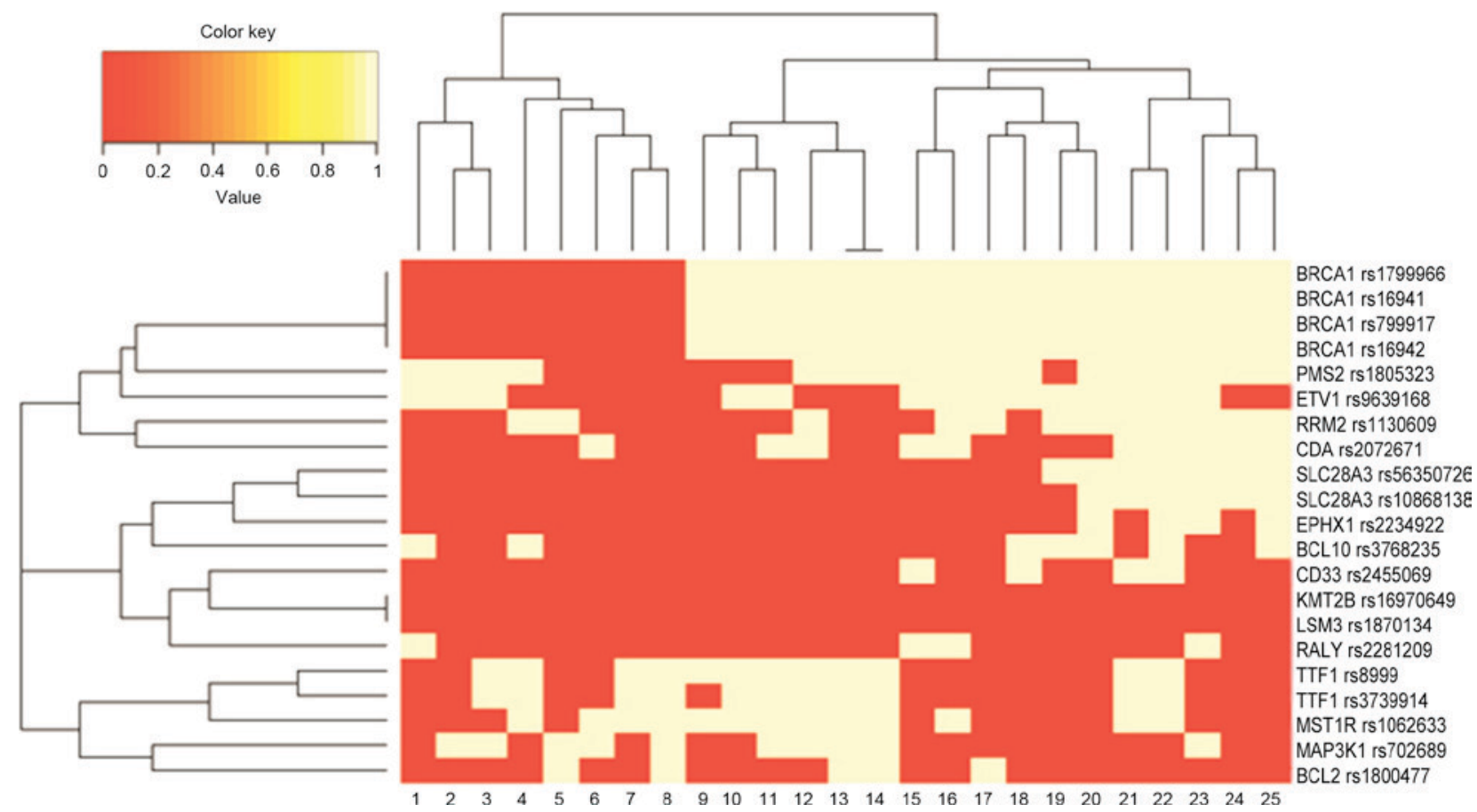

Figure 2. Clustering of correlations between single nucleotide polymorphisms and groups of patients with colorectal cancer. The color key indicates the correlation coefficient, with red being the most-associated. BRCA1, breast cancer 1; PMS2, PMS1 homolog 2; ETV1, ets variant 1; RRM2, ribonucleotide reductase regulators subunit M2; CDA, cytidine deaminase; SLC28A3, solute carrier family 28 member 3; EPHX1, epoxide hydrolase 1; BCL10, B cell lymphoma 10; CD33, Siglec-3; KMT2B, lysine methyltransferase 2B; LSM3, U6 small nuclear RNA-associated Sm-like protein 3; RALY, ribonucleoprotein particle-associated with lethal yellow; TTF1, thyroid transcription factor 1; MST1R, macrophage stimulating 1 receptor 1; MAP3K1, mitogen-activated protein 3 kinase 1; BCL2, B cell lymphoma 2.

number alterations, which accounted for $60 \%$ of all subjects. In the 1,503 candidate genes, 80 were identified to have CNVs. However, only one of the CNVs in the 80 genes (DDR1) was associated with lymph node metastasis in colorectal cancer, although this was not statistically significant $(\mathrm{P}=0.072)$.

Gene expression alteration in lymph node metastasis of colorectal cancer. The relative mRNA expression level of EGF in tumor tissues $(1.00 \pm 0.28)$ was significantly lower than in normal tissues $(4.89 \pm 1.56 ; \mathrm{P}<0.05)$. The relative mRNA expression level of NFKB1 in tumor tissues $(3.23 \pm 0.80)$ was significantly higher than in normal tissues $(1.25 \pm 0.25 ; \mathrm{P}<0.05)$.

\section{Discussion}

In the present study, 21 SNPs in 16 genes associated with lymph node metastasis of colorectal cancer were screened. Only $1 \mathrm{CNV}$ in the DDR1 gene was identified to be associated with lymph node metastasis in colorectal cancer, although this difference was not statistically significant. EGF and NFKB1 were abnormally expressed in colorectal tumor tissues.

EGF is a multi-functional growth factor; it is able to bind specific receptors on the cell surface and further induce signal transduction. In tumor cells, EGF may mediate proliferation by activating the EGF receptor pathway which leads to tumor cells survival and metastasis (18). Previous studies demonstrated that EGF is able to induce tumor metastasis through matrix metalloproteinases (19), tyrosine kinase PK2 (20), Podoplanin (21), Rictor binding protein (22), epithelial mesenchymal transition (23) and improving blood vessel (24) and lymph gland growth $(25,26)$. In the present study, the mRNA level of EGF was significantly upregulated in patients with lymph node metastasis, which indicated that EGF may be associated with lymph node metastasis in colorectal cancer. A previous study demonstrated that EGF is able to induce lymph gland metastasis by promoting lymph gland progression (19).

NFKB was initially identified in B lymphocytes, it is associated with a number of transcriptional processes by binding to promoter sites. NFKB is rarely mentioned as being associated with lymph node metastasis. The current study indicates that a significant upregulation of NFKB occurs during lymph node metastasis in colorectal cancer, indicating that NFKB may be associated with lymph node metastasis. The present study may provide a basis for further validation and identification of genetic markers.

In conclusion, the results of the present study indicate a number of potential genetic biomarkers associated with lymph node metastasis which may provide insight into early prognosis of colorectal cancer. However, a limitation of the present study was that the sample size was small. Therefore, a larger sample size is required for further validation of genetic biomarkers for colorectal cancer.

\section{Acknowledgements}

The present study was supported by the Project of Basic Research Plan Program of Shenzhen (grant no. JCYJ20160328161613864), the Innovation Program of Shenzhen (grant no. JCYJ20150330102720122), the Project of Shenzhen Basic Development Program (grant nos. JCYJ20120613171430264 and JCYJ20130329110928684), the Project of Shenzhen International Cooperation 
Foundation (grant nos. GJHZ20130412153906740 and GJHZ20160301163138685), the Nature Science Foundation of Guangdong (grant no. 2016A030313029) and the Project of Guangdong Scientific Plan Foundation (grant nos. 2013B021800097 and 2014A020212038).

\section{References}

1. American Cancer Society: Cancer facts and figures 2016 American Cancer Society, Atlanta, GA, 2016.

2. Ilic M and Ilic I: Colorectal cancer mortality trends in Serbia during 1991-2010: An age-period-cohort analysis and a joinpoint regression analysis. Chin J Cancer 35: 55, 2016.

3. Bishehsari F, Mahdavinia M, Vacca M, Malekzadeh R and Mariani-Costantini R: Epidemiological transition of colorectal cancer in developing countries: Environmental factors, molecular pathways, and opportunities for prevention. World J Gastroenterol 20: 6055-6072, 2014.

4. Abramson RG: Overview of targeted therapies for cancer. In: My Cancer Genome. https://www.mycancergenome.org/content/ molecular-medicine/overview-of-targeted-therapies-for-cancer/. Accessed July 29, 2016/

5. Gupta A, Kaur CD, Saraf S and Saraf S: Targeting of herbal bioactives through folate receptors: A novel concept to enhance intracellular drug delivery in cancer therapy. J Recept Signal Transduct Res 1-10, 2017.

6. Matsumoto K, Umitsu M, De Silva DM, Roy A and Bottaro DP: HGF-MET in cancer progression and biomarker discovery. Cancer Sci Jan 8, 2017 (Epub ahead of print).

7. Bozzi F, Mogavero A, Varinelli L, Belfiore A, Manenti G, Caccia C, Volpi CC, Beznoussenko GV, Milione M, Leoni V, et al: $\mathrm{MIF} / \mathrm{CD} 74$ axis is a target for novel therapies in colon carcinomatosis. J Exp Clin Cancer Res 36: 16, 2017.

8. Li X, Zhang G, Wang Y, Elgehama A, Sun Y, Li L, Gu Y, Guo W and $\mathrm{Xu} \mathrm{Q}$ : Loss of periplakin expression is associated with the tumorigenesis of colorectal carcinoma. Biomed Pharmacother 87: 366-374, 2017.

9. Chen W, Chen X and Lou R: Clinical study on lymph node metastasis of colorectal cancer. Chin J Cancer 19: 479-480, 2000 (in Chinese).

10. Grady WM and Carethers JM: Genomic and epigenetic instability in colorectal cancer pathogenesis. Gastroenterology 135: 1079-1099, 2008.

11. Sen S: Aneuploidy and cancer. Curr Opin Oncol 12: 82-88, 2000.

12. Pérez-Torras S, Vidal-Pla A, Cano-Soldado P, Huber-Ruano I, Mazo A and Pastor-Anglada M: Concentrative nucleoside transporter 1 (hCNT1) promotes phenotypic changes relevant to tumor biology in a translocation-independent manner. Cell Death Dis 4: e648, 2013.

13. Shivakumar BM, Rotti H, Vasudevan TG, Balakrishnan A, Chakrabarty S, Bhat G, Rao L, Pai CG and Satyamoorthy K: Copy number variations are progressively associated with the pathogenesis of colorectal cancer in ulcerative colitis. World J Gastroenterol 21: 616-622, 2015.
14. Chen Z, Liu Z, Deng X, Warden C, Li W and Garcia-Aguilar J: Chromosomal copy number alterations are associated with persistent lymph node metastasis after chemoradiation in locally advanced rectal cancer. Dis Colon Rectum 55: 677-685, 2012

15. Liang JW, Shi ZZ, Zhang TT, Hao JJ, Wang Z, Wang XM, Yang H, Wang MR, Zhou ZX and Zhang Y: Analysis of genomic aberrations associated with the clinicopathological parameters of rectal cancer by array-based comparative genomic hybridization. Oncol Rep 29: 1827-1834, 2013.

16. Sawada T, Yamamoto E, Suzuki H, Nojima M, Maruyama R, Shioi Y, Akasaka R, Kamimae S, Harada T, Ashida M, et al: Association between genomic alterations and metastatic behavior of colorectal cancer identified by array-based comparative genomic hybridization. Genes Chromosomes Cancer 52: 140-149, 2013.

17. Livak KJ and Schmittgen TD: Analysis of relative gene expression data using real-tie quantitative PCR and the 2(-Delta Delta C(T)) Method. Methods 25: 402-408, 2001

18. Maurer G, Tarkowski B and Baccarini M: Raf kinases in cancer-roles and therapeutic opportunities. Oncogene 30: 3477-3488, 2011.

19. Kajanne R, Miettinen P, Mehlem A, Leivonen SK, Birrer M, Foschi M, Kähäri VM and Leppä S: EGF-R regulates MMP function in fibroblasts through MAPK and AP-1 pathways. J Cell Physiol 212: 489-497, 2007.

20. Verma N, Keinan O, Selitrennik M, Karn T, Filipits M and Lev S: PYK2 sustains endosomal-derived receptor signalling and enhances epithelial-to-mesenchymal transition. Nat Commun 6: 6064,2015

21. Inoue H, Miyazaki Y, Kikuchi K, Yoshida N, Ide F, Ohmori Y, Tomomura A, Sakashita H and Kusama K: Podoplanin expression during dysplasia-carcinoma sequence in the oral cavity. Tumour Biol 33: 183-194, 2012.

22. Zhang F, Zhang X, Li M, Chen P, Zhang B, Guo H, Cao W, Wei X, Cao X, Hao X and Zhang N: mTOR complex component Rictor interacts with PKCzeta and regulates cancer cell metastasis. Cancer Res 70: 9360-9370, 2010.

23. Hardy KM, Booth BW, Hendrix MJ, Salomon DS and Strizzi L: ErbB/EGF signaling and EMT in mammary development and breast cancer. J Mammary Gland Biol Neoplasia 15: 191-199, 2010.

24. Kim HD, Meyer AS, Wagner JP, Alford SK, Wells A, Gertler FB and Lauffenburger DA: Signaling network state predicts twist-mediated effects on breast cell migration across diverse growth factor contexts. Mol Cell Proteomics 10: M111.008433, 2011.

25. Bracher A, Cardona AS, Tauber S, Fink AM, Steiner A, Pehamberger H, Niederleithner H, Petzelbauer P, Gröger M and Loewe R: Epidermal growth factor facilitates melanoma lymph node metastasis by influencing tumor lymphangiogenesis. J Invest Dermatol 133: 230-238, 2013.

26. Rovenská E: Importance of lymphangiogenesis and ultrastructure of lymphatic capillaries in metastasis of malignant melanoma. Vnitr Lek 60: 582-585, 2014. 